\title{
3D-based face segmentation using adaptive radius
}

\begin{abstract}
Face detection and segmentation is an important prerequisite step for many face related processes such as face recognition and facial expression recognition. A method that automatically segments the given faces images irrespective of their different sizes and orientations will not only ease the subsequent face analysis task but will as well enhances its performance. In this work, an adaptive radius based face segmentation method is presented. We utilised the face's intrinsic properties derived from Gaussian and mean curvature of the face surface to segment each face. The UPM-3DFE and Gavab 3D face databases were used in testing the new method. Visual inspection of the result indicated that the novel method can attained up to $99.23 \%$ segmentation accuracy.
\end{abstract}

Keyword: 3D face detection; Linear regression analysis; HK-classification 